\title{
Self-assembled pH-sensitive nanoparticles based on Ganoderma lucidum polysaccharide-methotrexate conjugates for the co-delivery of anti-tumor drugs
}

Dan Zheng, ${ }^{a}$ Jingyang Zhao ${ }^{a}$, Yucheng Li ${ }^{a}$, Liyu Zhu ${ }^{a}$, Mengchen Jin ${ }^{a}$, Luying Wang ${ }^{a}$, Jing Liu ${ }^{a}$, Jiandu Leia*, Zhonglong $L i^{b *}$

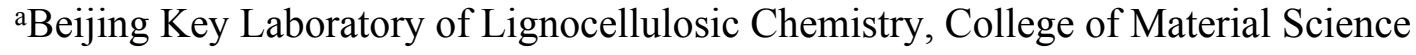
and Technology, Beijing Forestry University, NO.35 Tsinghua East Road, Haidian District Beijing, CN 100083, China

${ }^{b}$ Department of Acupuncture and Massage, Beijing Hospital, National Center of Gerontology, Institute of Geriatric Medicine, Chinese Academy of Medical Science, NO.1 Dongdan Dahua Road, Dongcheng District, Beijing, CN 100730, China

*Corresponding Author:

E-mail address: ljd2012@bjfu.edu.cn (Jiandu Lei), and lizhonglongbaby@163.com (Zhonglong Li) 


\section{Supporting Information (SI)}

SUPPORING FIGURES

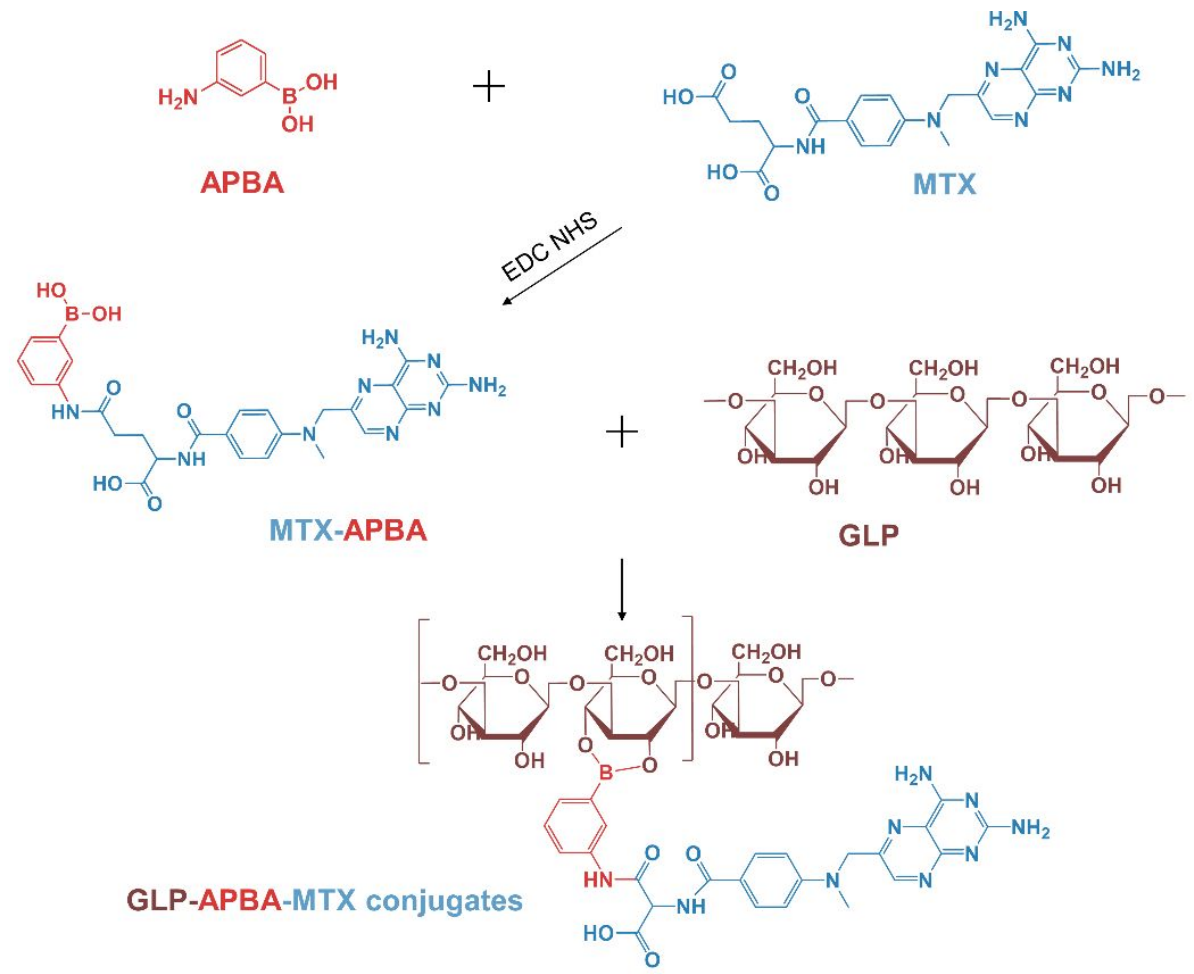

Supporting Figure S1. Graphical synthetic route of GLP-APBA-MTX conjugates 

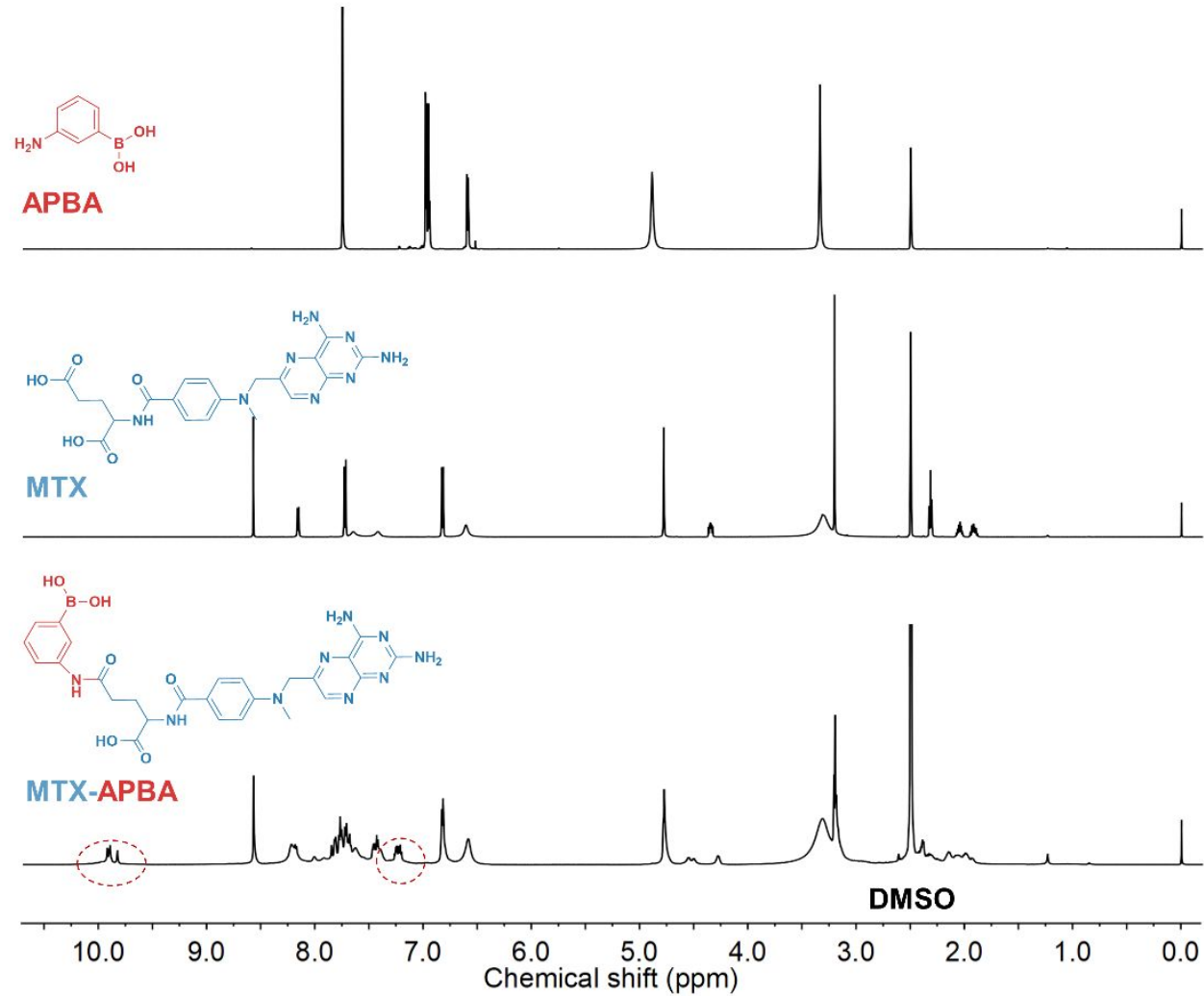

Supporting Figure S2. ${ }^{1} \mathrm{H}$ NMR spectra of APBA, MTX and MTX-APBA in DMSO 


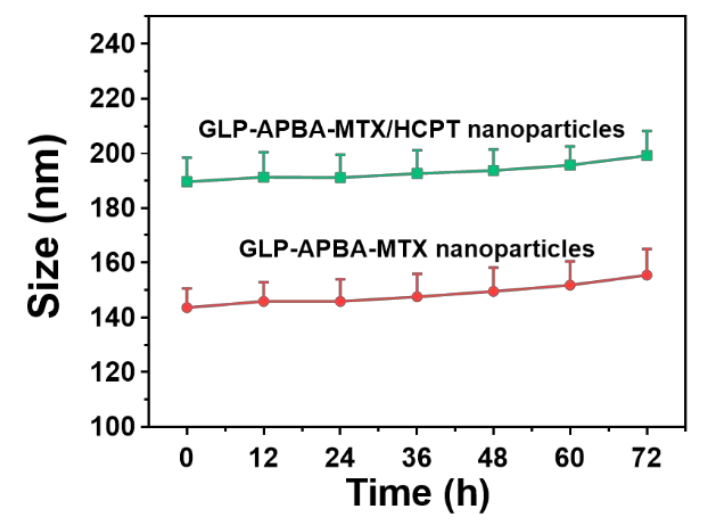

Supporting Figure S3. The stability of GLP-APBA-MTX nanoparticles and GLP-APBAMTX/HCPT nanoparticles under the different time 